Canadian University Music Review

Revue de musique des universités canadiennes

\title{
Luciano Berio. Entretiens avec Rossana Dalmonte. Traduits et présentés par Martin Kaltnenecker. Paris : Lattès, 1983, 188 pp.
}

\section{Gaëtan Martel}

Numéro 6, 1985

URI : https://id.erudit.org/iderudit/1014071ar

DOI : https://doi.org/10.7202/1014071ar

Aller au sommaire du numéro

Éditeur(s)

Canadian University Music Society / Société de musique des universités

canadiennes

ISSN

0710-0353 (imprimé)

2291-2436 (numérique)

Découvrir la revue

Citer ce compte rendu

Martel, G. (1985). Compte rendu de [Luciano Berio. Entretiens avec Rossana Dalmonte. Traduits et présentés par Martin Kaltnenecker. Paris : Lattès, 1983, 188 pp.] Canadian University Music Review / Revue de musique des universités canadiennes, (6), 329-334. https://doi.org/10.7202/1014071ar

(C) Canadian University Music Society / Société de musique des universités canadiennes, 1985
Ce document est protégé par la loi sur le droit d'auteur. L'utilisation des services d'Érudit (y compris la reproduction) est assujettie à sa politique d'utilisation que vous pouvez consulter en ligne.

https://apropos.erudit.org/fr/usagers/politique-dutilisation/ 
Le Discours musical est donc un manifeste, qui va constamment dans les exemples précis chercher les preuves de son argumentation: l'interprète reste toujours sur terre. Le grand public, l'amateur et le musicien y trouveront leur compte. Cependant je verrai là les limites du genre : rien dans l'ouvrage ne permet de prolonger la curiosité du lecteur. $\mathrm{Si}$ on passe sur le fait que Harnoncourt ne cite jamais les ouvrages fondamentaux publiés depuis un siècle sur l'interprétation de la musique ancienne, on peut regretter qu'aucune lecture plus spécialisée sur les rapports entre la musique et la rhétorique ne soit suggérée. On quitte donc le livre avec l'impression que l'auteur a tout découvert et que tout reste à faire. Les gens "du milieu " y trouveront de la frustration, les autres resteront un pied en l'air. Or la rhétorique étant une science complexe, ses rapports avec la musique demandant de prudents approfondissements : il est à craindre que le Discours musical ne provoque que de superficielles conversations de salon.

Mais après tout, le but est de "faire choc " dans le grand public. Quelque peu remise à sa place, la Beauté décorative de la musique laissera alors peut être le champ à la musique-discours. Harnoncourt espère bien qu'on découvrira alors que ce discours est encore le nôtre, qu'il nous est contemporain. A nous de savoir ce que nous avons à dire.

Jean-Pierre Pinson

LUCIANO BERIO. Entretiens avec Rossana Dalmonte. Traduits et présentés par Martin Kaltnenecker. Paris : Lattès, 1983, 188 pp.

Si un phénomène est particulièrement en vogue dans la littérature musicale, en cette seconde moitié du $\mathrm{XX}^{\mathrm{e}}$ siècle, c'est bien celui des entretiens avec les musiciens. Certains de ces entretiens sont même devenus des références comme un portrait (parmi plusieurs possibles) de personnalités à un moment de leur évolution. On songe ici naturellement au Par volonté et par hasard, ces entretiens de Célestin Deliège avec Pierre Boulez (un des premiers en date et qui remonte à 1972-1974), ou encore à ceux de Jonathan Cott avec Karlheinz Stockhausen ou Glenn Gould.

C'est dans la même collection que ces deux derniers ouvrages, "Musique et Musiciens ", que paraît ces entretiens avec Luciano Berio." Rossana Dalmonte, d'après les notes de la couverture de ce volume, " enseigne à l'université de Bologne. Critique musicale, elle collabore à différentes revues. Elle est l'auteur de nombreux essais et monographies. " On retiendra d'elle tout spécialement Il gesto della forma, Musica, Poesia, Teatro nello Opera di L. Berio, publié en 1981, en collaboration avec L. Lorenzini, L. Azzaroni et F. Frasdredi.

La traduction des Entretiens a été réalisé par Martin Kaltnenecker dans un français limpide, très facile d'accès et qui semble très fidèle à l'original. Par contre, sa préface est on ne peut plus douteuse et on se demande même qu'elle en est la pertinence, exception faite de n'être qu'une 
forme d'exhibitionnisme peu subtil. En effet, véritable logorrhée où l'auteur se complait à multiplier les citations (veut-il faire montre de sa "culture ", de son " savoir encyclopédique " ?), elle ne nous apporte à peu près rien par rapport au contenu de ces entretiens. Alain, Balzac, Barthes, Butor, Char, Horace, Nietzsche, Valéry ... en huit pages! On a droit à une belle "salade d'idées " : "La lecture est un dépecement, une mort, un viol, elle est masculine (?) et destructrice (?) » (p. 5). Et plus loin : "Le monde de Berio, tel qu'il le décrit, a un sens, c'est-à-dire une direction ... » (p. 14). Il n'a pas qu'un sens, il a des sens : une direction et une signification. Mais l'essentiel de cet ouvrage, ce sont les entretiens comme tel. Six parties, de proportions inégales, constituent le tout.

La première partie, "Ce qu'est la musique aujourd'hui ", est une introduction au sujet, par le biais d'une définition de ce qu'est, pour Berio, la musique. Avec cette question, probablement une des plus complexes qui soit, on découvre immédiatement le ton général de ces discussions. Berio possède une vision ouverte des choses, généreuse et humaniste, un respect constant pour toutes les formes de métiers, ce qui se retrouve également dans ses oeuvres, toujours conçues en fonction des possibilités réelles de l'intrumentiste. Ses convictions politiques (il vote communiste) se reflètent constamment dans son discours : il parle de contrat social des réceptions et productions en musique ; d'éducation musicale qui devrait être démocratisée, alors qu'elle est pensée en fonction des besoins d'une élite ; et du " sens de l'histoire " que l'on retrouve en musique comme partout ailleurs (attitude en partie marxiste).

Ce qu'est la musique pour Berio ? " La musique est tout ce que l'on écoute avec l'intention d'écouter de la musique " (p. 23) - une définition souple s'il en est - " harmonisant et transformant la nature et la culture, elle est à la fois un processus pratique et empirique tout en atteignant les couches profondes de notre être " (p. 25). Par la suite, il tente de poser ce qu'est pour lui une stratégie de réception. Tout aussi flexible que sa définition précédente, elle n'est qu'une ouverture qui vise à la multiplicité des points de vue. Pour lui, « il y a des façons plus compliquées et d'autres plus simples d'écouter la musique " mais "tout le monde, à sa façon, comprend la musique » (p. 31).

Un aspect également intéressant de cette première partie est qu'elle contient en germe les cinq suivantes. Il y glisse quelques mots sur la pratique de la musique de chambre chez son père, dans sa jeunesse, et de la première audition d'un disque (de jazz New Orleans) en 1943, renseignements biographiques qui généreront les deuxième et troisième parties.

À la toute fin de ce chapitre il amorce une réflexion sur le retour des jeunes vers une "pratique musicale", ce retour vers une "musique autre" (p. 53). Cette insistance sur le "faire musical» (p. 54) se retrouvera dans la quatrième partie, "Les jeunes musiciens".

Enfin, il parle aussi de son projet de traité d'instrumentation idéal -utopique et qui ne vit jamais le jour — en collaboration avec Boulez, à 
l'époque où il travaillait à l'I.R.C.A.M. Boulez, pour lequel il entretient un profond respect tout au long de ces conversations, demeure pour lui l'écrivain sur la musique le plus significatif de notre époque grâce à sa " cohérence et transparence stupéfiantes " et sa facilité à réaliser des bilans de l'histoire, toujours convaincants.

À l'autre extrémité, il place Adorno, auquel il reproche ses attitudes "snob, moraliste et de généralisateur suspect", sa "conception monolitique " des faits étant toutefois doublée d'un " grand aphoriste ".

Nous retiendrons de ce chapitre un dernier point concernant plus spécifiquement la musicologie, ses origines et son évaluation critique. C'est toujours Dalmonte qui l'interroge :

D. - Il serait intéressant de comprendre pourquoi on a commencé, à partir de la mort de Beethoven, à parler et à écrire tellement sur la musique.

B. - Peut-être parce que la musique a cessé d'être une activité objective, destinée à assurer des fonctions spécifiques, pour devenir, par ses intentions du moins, le véhicule des idées et d'une expressivité personnelles. " (p. 19) ( ...). Une distance s'est créée entre idée et pratique musicale, et le musicien qui en était conscient a dû l'expliquer et la réduire, pour un public qui payait et voulait réentendre une symphonie, non seulement l'écouter une fois $(\ldots)$. Le compositeur a commencé à parler de son travail et de ses visions dès l'instant où il s'est détaché de la pratique directe de la musique ..." (p. 20)

"... Parler de la musique peut faire partie du processus créatif de la composition. Nous vivons dans un monde de paroles écrites, et tout ce qui n'est pas écrit ou impossible à écrire est souvent déformé ou même n'existe pas. Il n'est pas rare en fait qu'un musicien soutienne étourdiment que telle musique extra-européenne est "improvisée " simplement parce qu'elle n'est pas notée. De plus en plus souvent je lis des écrits sur la musique qui n'ont rien à voir avec une expérience musicale effective, réelle, ni avec un projet musical : ce sont des paraphrases verbales de virtualités musicales. »(p. 44)

La seconde partie, " D'Oneglia aux U.S.A. », retrace tout l'apprentissage musical de Berio. Cette période s'étale des premiers contacts avec la musique dûs à son père et son grand-père, tous deux musiciens, jusqu'à son départ pour les U.S.A. au tout début des années 1950. D'Oneglia, sa patrie natale, il se remémore les pratiques de musique de chambre en famille et cette première audition radiophonique de La Bohème qui le bouleversa totalement aux environs de sa treizième année. Par après, ce fut le conservatoire de Milan et ces rencontres fondamentales avec Bruno Maderna, Henri Pousseur et Luigi Dallapiccola aux U.S.A. C'est une vision très lucide qu'il a de ce pays :

«... J'ai la certitude que si cette société est opulente et équipée des instruments culturels les plus évolués (les cerveaux des corps académiques dans les grandes universités ... (...), c'est parce que quelqu'un en fait les frais quelque part sur la planète. " (p. 76) 
De plus, pour lui,

" l'avant-garde musicale américaine (est) désormais réduite à une sorte de maniérisme - (et) ne sert à rien ; on se débat dans une société - qui est en partie aussi la nôtre - où " le marché récupère même le refus de marché. » (p. 77)

C'est un véritable fondu enchaîné qui relie le chapitre précédent à "L'expérience sérielle» puisqu'on ne fait que poursuivre l'évolution chronologique de Berio. Nous sommes au début de cette ère bouillonnante de créations, les années 1950, avec ces enfants terribles du temps qu'étaient Boulez, Cage et Stockhausen. C'est Darmstadt naturellement, mais également le détachement de cette capitale de la musique contemporaine pour Berio, grâce à ces oeuvres majeures : Nones, Allelujah I et II. Cette section se termine avec un commentaire sur la pensée minimaliste en général, et les U.S.A. :

"Souvent, en Amérique surtout, le besoin de séparer, de faire remarquer et de rendre reconnaissables (sur le plan élémentaire du comportement) comme entités autonomes le matériau et la matière, cache une attitude réductrice, empruntée en partie au marché de la peinture. " (p. 98)

"Les jeunes musiciens ", partie la plus courte du volume (16 pages), nous apparait comme une transition vers la seconde section la plus importante de ce livre, "Le métier de compositeur ". Il y est bien sûr question de certaines tendances récentes - improvisation surtout, avec l'exemple du groupe New Phonic Art et celle, qualifiée de " curieuse et pathétique ", de " reconquête de la mélodie " (p. 107) —, mais on observe déjà des commentaires sur le métier de compositeur dans son essence même. Pour Berio, les bases sont simples et évidentes : on doit réaliser un grand nombre de travaux d'écriture et, plus précisément, de contrepoint traditionnel.

Cette approche contrapuntique de la musique se retrouve tout naturellement dans la série d'oeuvres probablement la plus connue de Berio : les Sequenza. Dans le chapitre V, il expose toutes les diverses caractéristiques analytiques de ces pièces et de la Sinfonia. Les éléments unificateurs de ces Sequenza sont au nombre de cinq : 1) ce sont presque tous des "suites de séquences - d'où leurs noms - , de champs harmoniques $(\ldots)$ dont surgissent, avec un maximum de caractérisation, les autres fonctions musicales" (p. 130) ; 2) la virtuosité y est présente partout ; 3) l'utilisation maximale des moyens techniques naturels des instruments ; 4) " le contrôle constant du parcours harmonique et de la densité mélodique " (p. 133) ; 5) l'intention de "préciser et de développer mélodiquement un discours essentiellement harmonique et de suggérer, surtout dans le cas des instruments monodiques, une écoute de type polyphonique. " (p. 129-130)

C'est lorsqu'il parle plus précisément de la Sequenza III que l'on constate tout l'intérêt que porte Berio à la dramaturgie et à la théâtralité de 
la musique (cf. Kagel). De plus - on peut faire ici le lien avec Puccini ${ }^{2}$ -cette Sequenza " assimile musicalement beaucoup d'aspects de la vocalité quotidienne ... " (p. 126)

"En marge des notes ", le dernier chapitre, n'est finalement qu'une coda où Berio rappelle l'importance, pour la création musicale, d'accorder ou d'harmoniser la composition avec un "sens de l'histoire", en conjonction avec les moyens technologiques de notre siècle. Il rend hommage à tout le travail de recherche et de créativité de pointe dans le domaine électro-acoustique et informatique, qu'effectue Pierre Boulez et son équipe à l'I.R.C.A.M. Il faut avant tout, déclare-t-il,

" être toujours en mesure d'offrir - à ceux qui viendront à notre rencontre, qi sait comment et qui sait d'où - des choses vraies et historiquement responsables, et non pas des choses feintes et sans mémoire " (p. 188)

D'où, tout au long du livre, une attention fondamentale aux problèmes de l'écriture et du lien entre le matériau et l'invention. ${ }^{3}$

Ce livre fascinera non seulement tous les passionnés de Berio qui le découvriront dans sa quotidienneté, mais également tous les simples amoureux de musique. Car Berio n'adopte aucun compromis dans son discours, toujours d'une chaleur bien latine, aussi bien envers ses amitiés indéniables (Pousseur, Maderna et, - histoire d'une vie - Cathy Berberian) que pour ses " ennemis " qu'il ne ménage pas : Mascagni, « le troglodyte» ; Eisler, "le débile musical» ; "l'absurbité musicale désespérée de Morton Feldman "; " les vieux papiers tue-mouches de Cage "; « le cadavre de Bellini que l'on devrait broyer » (!). Ces pages sont également fondamentales pour quiconque veut (re) dévouvrir l'univers musical italien de notre siècle, moins bien connu qu'on ne l'imagine, à travers ses critiques (tel Lele d'Amico), ses écrivains sur la musique (Massimo Mila), ou ses musiciens méconnus (Paribeni, Ghedini ...). Cet ouvrage offre même des possibilités prospectives pour la recherche musicologique : Berio suggère une étude sociologique de l'apparition, au XIXe siècle, des écrits des compositeurs devenus "non-exécutants", comme reflet de la distinction travail manuel/travail intellectuel. Il constate également que l'histoire de la transcription en musique demeure entièrement à (d)écrire.

Les seules réserves que nous émettons portent sur la présentation matérielle. Il aurait été souhaitable, pour une plus grande intelligibilité et articulation du propos, de subdiviser les différentes parties avec des intertitres. Nous trouvons également malheureux d'avoir omis la date exacte de ces entretiens.

Mais somme toute, ce livre demeure un véritable microcosme musical où l'on retrouve une abondance d'informations à tous les niveaux (théorique, esthétique, historique), ce qui demeure la caractéristique la plus intéressante de cet ouvrage. Assurément, une des meilleures publications disponible dans ce type de collection. 


\section{NOTES}

1. Notons que le dernier entretien avec Berio publié en français figure dans le " dossier Berio " du premier numéro de Contrechamps, tout nouveau périodique spécialisé dans la musique actuelle. L'ensemble de ce dossier est, du reste, excellent puisqu'il intègre analyses, écrits de Berio et un très précieux appareil critique (discographie, bibliographie, etc . . . ). Réalisée par Philippe Albèra et Jacques Demierre (1983), la discussion avec Berio (pp. 60 à 66) rappelle des points qu'on retrouve dans les entretiens avec Dalmonte : 1) les relations littéraires, linguistiques, musicales avec des auteurs comme Calvino, Eco et Sanguinetti ; 2) son intérêt pour la " pluralité musicale " (il parle d' " anthologie de styles et de techniques diverses" - p.61) telle l'intégration des techniques d' "écriture " non occidentales, celle des Banda-Linda par exemple ; 3) son goût du lyrisme, de la musicalité vocale et du théâtre en général, dans sa dernière création : Un re in Ascolto.

2. "Puccini a introduit dans le théâtre musical le temps quotidien, la mobilité psychologique de la vie de tous les jours " (p. 65).

3. Voir en particulier aux pages 84, 85, 89, 92, 115, 138, 141, 162 et 175.

Anton F. Kolstee. Bella Coola Indian Music: A Study of the Interaction between Northwest Coast Indian Structures and their Functional Context. 2 vols. Service Canadien d'ethnologie, dossier No. 83. Ottawa : Musée National de l'Homme, 1982. x, 274 pp., musique, illus., bibliographie.

Le problème de l'interaction entre les structures musicales et la culture est une composante fondamentale de l'ethnomusicologie contemporaine. Dans son volume, Bella Coola Indian Music, Anton Kolstee discute du lien entre les chants des indiens Bella Coola et leur contexte fonctionnel. Il nous présente 73 transcriptions de sources variées, la plupart provenant de son terrain (Colombie britannique, 1975), tout en avouant les limites de son étude, étant donné qu'aucun des chanteurs actuel n'est compositeur et que de nombreux aspects de leur culture sont perdus. L'auteur ajoute l'information ethnographique provenant de collectes effectuées depuis le XIXe siècle, s'appuyant en particulier sur le travail de Thomas F. McIlwraith (1948).

L'objectif principal de cet ouvrage est de montrer, partout où c'est possible, les déterminants sociaux de la musique des Bella Coola par un examen de la dialectique entre les facteurs extra-musicaux (par exemple la danse et l'organisation de son exécution) et les caractéristiques structurales de la musique.

Kolstee répartit les chants en deux groupes principaux : les cérémoniels et les non-cérémoniels. Les membres de deux sociétés secrètes, la Sisawk et la Kusiyut, héritent, commandent composent ou reçoivent des tribus voisines toutes les chansons cérémonielles de leur tribu. Les chansons de coiffure (au nombre de 13) et les chansons de deuil 